\title{
Genetic Variants in the Region Harbouring IL2/IL21 Associated to Ulcerative Colitis
}

\author{
Eleonora A.M. Festen ${ }^{1,2}$, Philippe Goyette ${ }^{3}$, Regan Scott ${ }^{4}$, Vito Annese ${ }^{5}$, Alexandra \\ Zhernakova $^{6}$, Jing Lian ${ }^{3}$, Céline Lefèbvre ${ }^{3}$, Steven R. Brant ${ }^{7,8}$, Judy H. Cho ${ }^{9}$, Mark S. \\ Silverberg ${ }^{10}$, Kent D. Taylor ${ }^{11}$, Dirk J. De Jong ${ }^{12}$, Pieter C. Stokkers ${ }^{13}$, Dermot \\ McGovern ${ }^{11}$, Orazio Palmieri ${ }^{5}$, Jean-Paul Achkar ${ }^{14}$, Ramnik J. Xavier ${ }^{15}$, Mark J. Daly 16,17, \\ Richard H. Duerr ${ }^{4,18}$, Cisca Wijmenga ${ }^{2}$, Rinse K. Weersma ${ }^{1}, \S$, and John D. Rioux ${ }^{3, \S}$
}

${ }^{1}$ Department of Gastroenterology and Hepatology, University Medical Center Groningen, University of Groningen, Groningen, the Netherlands ${ }^{2}$ Department of Genetics, University Medical Center Groningen and University of Groningen, Groningen, the Netherlands ${ }^{3}$ Laboratory in Genetics and Genomic Medicine of Inflammation, Montreal Heart Institute Université de Montréal, Montreal, Canada ${ }^{4}$ Division of Gastroenterology, Hepatology and Nutrition, Department of Medicine, University of Pittsburgh School of Medicine, UPMC-PUH, Mezzanine Level, C-Wing, 200, Lothrop Street, Pittsburgh, PA 15261, USA. ${ }^{5}$ UU.OO. Gastroenterologia ed Endoscopia Digestiva, Ospedale "Casa Sollievo della Sofferenza", IRCCS, San Giovanni Rotondo, Italy. ${ }^{6}$ Complex Genetics Section, Department of Medical Genetics, University Medical Center, Utrecht, Utrecht, the Netherlands ${ }^{7}$ Harvey M. and Lyn P. Meyerhoff Inflammatory Bowel Disease Center, Department of Medicine, Johns Hopkins University, 1503 East Jefferson Street, Baltimore, Maryland 21231, USA ${ }^{8}$ Department of Epidemiology, Bloomberg School of Public Health, Johns Hopkins University, 615 E. Wolfe Street, Baltimore, Maryland 21205, USA ${ }^{9}$ Departments of Medicine and Genetics, Division of Gastroenterology, Inflammatory Bowel Disease (IBD) Center, Yale University, 300 Cedar Street, New Haven, Connecticut 06519, USA ${ }^{10}$ Mount Sinai Hospital IBD Centre, University of Toronto, 441-600 University Avenue, Toronto, Ontario M5G 1X5, Canada ${ }^{11}$ Medical Genetics Institute and Inflammatory Bowel Disease (IBD) Center, Cedars-Sinai Medical Center, 8700 W. Beverly Blvd., Los Angeles, California 90048, USA ${ }^{12}$ Department of Gastroenterology and Hepatology, Radboud University Nijmegen Medical Centre, Nijmegen, the Netherlands ${ }^{13}$ Department of Gastroenterology and Hepatology, Academic Medical Center, Amsterdam, the Netherlands ${ }^{14}$ Center for Inflammatory Bowel Disease, Department of Gastroenterology \& Hepatology, Cleveland Clinic, Cleveland, Ohio, USA; ${ }^{15}$ Center for Computational and Integrative Biology and Gastrointestinal Unit, Massachusetts General Hospital, Harvard Medical School, 185 Cambridge Street, Boston, Massachusetts 02114, USA ${ }^{16}$ Center for Human Genetic Research, Massachusetts General Hospital, Harvard Medical School, 185 Cambridge Street, Boston, Massachusetts 02114, USA ${ }^{17}$ Molecular Biology

Correspondence to: Rinse K. Weersma, Department of Gastroenterology and Hepatology, University of Groningen and University Medical Center Groningen, PO Box 30001; 9700 RB Groningen; the Netherlands, Tel.: (+31) 50 3613354, Fax: (+31) 50 3619306, R.K.Weersma@int.umcg.nl, John D. Rioux, Ph.D. Université de Montréal \& Montreal Heart Institute, Tel.: (514) 376-3330 ext. 3741 , Fax: (514) 593-2539, john.david.rioux@umontreal.ca.

$\S$ These authors contributed equally to this work.

The work was performed at address, Department of Genetics, University Medical Center Groningen and University of Groningen, Groningen, the Netherlands and Laboratory in Genetics and Genomic Medicine of Inflammation, Montreal Heart Institute Université de Montréal, Montreal, Canada.

Authors' disclosure: No authors have a conflict of interest to declare.

The Corresponding Author has the right to grant on behalf of all authors, and does grant on behalf of all authors, an exclusive licence (or non-exclusive for government employees) on a worldwide basis to the BMJ Publishing Group Ltd and its Licensees to permit this article (if accepted) to be published in Gut and any other BMJPGL products to exploit 75 all subsidiary rights, as set out in our licence (http://gut.bmj.com/ifora/licence.pdf). 
Department, Massachusetts General Hospital, Harvard Medical School, 185 Cambridge Street, Boston, Massachusetts 02114, USA ${ }^{18}$ Department of Human Genetics, Graduate School of Public Health, University of Pittsburgh, 130 Desoto Street, Pittsburgh, Pennsylvania 15261, USA;

\section{Abstract}

Objectives-Genetic susceptibility is known to play a large part in the predisposition to the inflammatory bowel diseases (IBD) known as Crohn's disease (CD) and ulcerative colitis (UC). The IL2/IL21 locus on 4q27 is known to be a common risk locus for inflammatory disease (shown in celiac disease, type 1 diabetes, rheumatoid arthritis, systemic lupus erythematosus and psoriasis), while the roles that IL2 and IL21 play in the immune response also make them attractive candidates for inflammatory bowel disease. Our objective was to test for association between the IL2/IL21 locus and the inflammatory bowel diseases.

Methods-The four single nucleotide polymorphisms (SNPs) in the IL2/IL21 locus most associated to celiac disease were genotyped in 1590 IBD cases and 929 controls from the Netherlands, and then replicated in a North American cohort (2387 cases and 1266 controls) and an Italian cohort (805 cases and 421 controls), yielding a total of 4782 cases ( $3194 \mathrm{UC}, 1588 \mathrm{CD}$ ) and 2616 controls. Allelic association testing and a pooled analysis using a Cochran-Mantel-Haenszel test were performed.

Results-All four SNPs were strongly associated with UC in all three cohorts and reached genomewide significance in the pooled analysis ( $r 13151961 \mathrm{p}=1.35 \times 10^{-10}$, rs $13119723 \mathrm{p}=8.60 \times 10^{-8}$, rs6840978 $\left.\mathrm{p}=3.07 \times 10^{-8}, \mathrm{rs} 6822844 \mathrm{p}=2.77 \times 10^{-9}\right)$. We also found a moderate association with $\mathrm{CD}$ in the pooled analysis ( $\mathrm{p}$ value range $0.0016-9.86 \times 10^{-5}$ ).

Conclusions-We found a strong association for the IL2/IL21 locus with UC, which also confirms it as a general susceptibility locus for inflammatory disease.

\section{Keywords}

Inflammatory bowel disease; ulcerative colitis; Crohn's disease; IL2; IL21

\section{Introduction}

Inflammatory bowel diseases (IBD) are the most common chronic inflammatory diseases in the Western world after rheumatoid arthritis, with an incidence of about 40 per 100,000 in North America and Western Europe.[1] Ulcerative colitis (UC) and Crohn's disease (CD) are the two main types of IBD, both characterized by recurring inflammation of the digestive tract. In CD the inflammation can occur throughout the gastrointestinal tract, most commonly affecting the terminal part of the small intestine and causing weight loss and abdominal pain. In UC the disease is confined to the colon and patients usually present with bloody diarrhoea and abdominal cramping.[2]

Genetic susceptibility plays an important role in the pathogenesis of IBD. CD and UC are complex diseases with numerous genetic and environmental factors leading to disease. Epidemiological studies suggest stronger heritability in CD as compared to UC.[1] Many genetic factors contributing to $\mathrm{CD}$ pathogenesis have been identified during the last decade. There are currently more than 30 genes or loci associated with $\mathrm{CD}$, the majority having been identified since the introduction of genome-wide association studies.[3] Far fewer have been found for UC. Recently the first genome-wide association study in UC was published identifying several new loci and another genome-wide association study will be published shortly.[4,5] 
Genetic studies have also shown that susceptibility genes are commonly shared between inflammatory diseases. For example, the IL2/IL2Ilocus on chromosome 4q27 has been shown to be associated with celiac disease, type 1 diabetes (T1D), Grave's disease (GD), systemic lupus erythematosus (SLE), rheumatoid arthritis (RA), and psoriasis (PS).[6-11] Interestingly, there appears to be at least two independent association signals in this region, one conferring increased risk to disease, while the other a protective effect.[8,11]

There are several reasons why the IL2/IL21 locus could also represent an interesting locus for IBD. Firstly, a number of shared autoimmune and inflammatory genes show an association to IBD: $I L 12 B$, for example, is associated to psoriasis,[10] systemic lupus erythematosus,[12] asthma[13] and both forms of IBD[14], while ILI8RAP was found to be associated with both celiac disease[15] and IBD.[16] IL2/IL2I is another shared inflammatory locus, and both $I L 2$ and $I L 21$ are attractive functional candidate genes for association to IBD. An overexpression of IL21 in inflamed regions of bowel of IBD patients has been reported.[17] This overexpression is most marked in $\mathrm{CD}$, but a significant overexpression compared to that in diverticular disease and healthy controls is also present in UC.[17] Finally, $I l 2^{-/-}$mice develop inflammatory bowel disease most reminiscent of UC.[18]

Given all these observations, and that IBD and celiac disease are chronic inflammatory diseases of the gut, we were specifically interested in testing whether the IL2/IL21 region variants identified in the celiac GWA studies also have a role to play in IBD. This was achieved via a case-control association study with a three-stage design in a large cohort of IBD patients. In addition, we performed genotype-phenotype analysis to identify association with specific subsets of IBD. Our data unequivocally show that the IL2/IL2I locus is strongly associated to UC. We confirmed this finding in multiple IBD populations.

\section{Methods}

\section{Subjects}

For the first phase, the cases consisted of a cohort of 1590 IBD patients (777 CD and $813 \mathrm{UC}$ ) collected from the outpatient clinics of the Departments of Gastroenterology and Hepatology at the Amsterdam Medical Center $(\mathrm{n}=732)$, the Radboud University Medical Centre, Nijmegen $(n=273)$, and the University Medical Center Groningen, the Netherlands $(n=585)$. [19] The control cohort consisted of 929 healthy Dutch individuals collected from blood donors.[6]

To replicate the findings from the first phase two independent cohorts were examined. The first replication cohort consists of an IBD case - control cohort (2387 cases of which $654 \mathrm{CD}$ and 1733 UC, and 1266 controls) collected through the North American NIDDK IBD Genetics Consortium (IBDGC) as described previously.[20,21] Cases and geographically matched controls were ascertained through the University of Montreal, Cedars-Sinai Medical Center, Johns Hopkins University, University of Chicago, University of Pittsburgh, and the University of Toronto Genetics Research Centers (GRCs). This NIDDK-IBDGC IBD cohort contained five related pairs of cases between UC and CD samples. All cases were included in the subphenotype analysis, but in the IBD analysis one member of each pair (five cases) was removed. The second replication cohort consists of an Italian IBD case - control cohort (805 cases of which $157 \mathrm{CD}$ and $648 \mathrm{UC}$, and 421 controls) collected at the S. Giovanni Rotondo "CSS" (SGRC) Hospital in Italy. This cohort has previously been used and characterized in several association reports from our group. [22,23] A f0ourth cohort consisting of 398 cases and 418 controls from the US of Jewish descent was also included; this cohort was also collected by the NIDDKIBDGC and has previously been characterized.[20,21]

All patients and controls were of European Caucasian descent. The diagnosis of IBD required (a) one or more symptoms of diarrhoea, rectal bleeding, abdominal pain, fever, or complicated 
perianal disease, (b) occurrence of symptoms on two or more occasions separated by at least 8 weeks or ongoing symptoms of at least 6 weeks duration, and (c) objective evidence of inflammation from radiologic, endoscopic, and histopathologic evaluation. All affected subjects fulfil clinical criteria for IBD. For CD patients, phenotypic details were registered according to the Vienna classification. However, perianal disease was scored as an independent variable and not included in the group with penetrating disease behaviour. For UC patients, phenotypes were described according to age of onset, maximum extent of disease (proctitis, left-sided, or extensive), necessity of colectomy, and the occurrence of malignancy and extraintestinal manifestations. A summary of the phenotype information available for each cohort can be found in supplementary table 1 (CD) and supplementary table 2 (UC).

In all cases, informed consent was obtained using protocols approved by the local institutional review board in all participating institutions. All DNA samples and data in this study were denominalized.

\section{Genotyping}

We analyzed the four most strongly associated SNPs in $I L 2 / I L 21$ found by Van Heel et al.: rs6822844, rs13151961, rs13119723 and rs6840978.[6] Genotyping of the Dutch cohort was performed using TaqMan technology, while SNP genotyping assays for PCR were supplied by Applied Biosystems, as described.[6] The patient and control DNA samples were processed in 384-well plates and each plate also contained 16 genotyping controls (4 duplicates of $4 \mathrm{CEU}$ DNA).

Genotyping of 1577 samples from the North American IBD cohort was performed using primer extension chemistry and mass spectrometric analysis (iPlex assay, Sequenom, San Diego, CA) on the Sequenom MassArray. This was performed at the Laboratory for Genetics and Genomic Medicine of Inflammation (www.inflammgen.org) of the Université de Montréal and at The University of Pittsburgh. Data from an additional set of 2917 North American IBD samples was also obtained from genotyping on Illumina HumanHap300 or HumanHap550 Genotyping BeadChips (Illumina, Inc., San Diego, CA, ) as was previously reported in the IBDGC's CD and UC GWAS studies.[5,21]

Genotyping for the Italian cohort was also performed at the Laboratory for Genetics and Genomic Medicine of Inflammation, using primer extension chemistry and mass spectrometric analysis on the Sequenom MassArray. The patient and control DNA samples were again processed in 384-well plates and each plate also contained 16 genotyping controls 4 duplicates of 4 CEU DNA). All SNPs were validated and we obtained $>99.9 \%$ concordance between our genotype data and the CEU data available from HapMap.

\section{Statistical analysis}

Hardy-Weinberg equilibrium was tested by comparing the expected and observed genotypes in a $2 \times 3$ chi-squared $\left(\chi^{2}\right)$ table. Controls did not show deviation from HWE ( $P$ value (HWE) $>0.001)$. Differences in allele and genotype distribution in the cases and controls of the individual cohorts were tested for significance by the $\chi^{2}$ test. Analysis for association between genotype and subphenotypes were also performed with the $\chi^{2}$ test. A significant threshold for $P$ values was determined at $P<0.05$. Odds ratios (ORs) were calculated and the confidence intervals (CIs) were approximated using Woolf's method with Haldane's correction. Power calculations were performed using the online Genetic Power Calculator by Shaun Purcell (http://pngu.mgh.harvard.edu/ purcell/gpc/).[24] Combined analysis of the different cohorts was performed by Cochran-Mantel-Haenszel meta-analysis. 


\section{Results}

Initially the rs 13151961, rs13119723, rs6840978 and rs6822844 SNPs were tested in 1590 Dutch patients ( $777 \mathrm{CD}$ patients and 813 UC patients) and 929 healthy controls. The minor alleles of all four SNPs tested were associated with IBD with a $P$ value range between 0.00093 and 0.00039 and an OR between 0.76 and 0.78 . This association was even stronger in the UC subgroup of the cohort ( $P$ value range $0.00038-0.00001$ and OR range $0.71-0.67)$. In the CD subgroup, the rs13119723 SNP was borderline significant with a $P$ value of 0.0327 , while only a trend towards association was observed for the other SNPs. This indicated that the association of the IL2/IL21 locus with IBD was coming predominantly from the UC subgroup. The results are shown in table 1

To replicate these findings we studied two independent cohorts. In the North American cohort (2387 IBD cases (654 CD and $1733 \mathrm{UC}$ ) and 1266 controls) we observed association with the same alleles of all SNPs in IBD (P value range $0.0011-0.0003$ and OR range $0.77-0.81$ ). As in the original cohort, this effect was strongest in the UC subgroup of the cohort ( $\mathrm{P}$ value range $0.0046-0.0004$ and OR range $0.77-0.81$ ). In the CD subgroup of the North American cohort a moderate association with the same alleles was also observed (P value range $0.0123-$ 0.0011). Testing of all four SNPs in the Italian cohort (805 IBD cases $157 \mathrm{CD}, 648 \mathrm{UC}$ ) and 421 controls) showed the same strong association of the minor alleles in UC as seen in the original cohort, with a P value range between 0.0123 and 0.0002 and an OR range between 0.75 and 0.62 . The $\mathrm{CD}$ subgroup of the Italian cohort showed only a trend towards association with the same alleles, which was not significant with a $P$ value range between 0.3495 and 0.0873 . The results are shown in table 1

A Cochran-Mantel-Haenszel meta-analysis of the results from all three cohorts showed a very convincing association of all IL2/IL21 SNPs in IBD (P value range $7.45 \times 10^{-6}-1.41 \times 10^{-9}$ ). In UC this effect also reached genome-wide significance with a $\mathrm{P}$ value of $3.07 \times 10^{-8}$ for rs 6840978 and a $P$ value of $1.35 \times 10^{-10}$ for rs 13151961 . The meta-analysis showed a moderate association with $\mathrm{CD}$ for all four SNPs to the same alleles ( $\mathrm{P}$ value range $0.0016-9.86 \times 10^{-5}$ ).

The fourth cohort consisting of patients with a Jewish background was analyzed separately, these results are depicted in table 2 . We did not find a significant association between any of the SNPs and CD in this cohort. We were reluctant to add this cohort to the meta analysis for all CD patients because of the large discrepancy in minor allele frequency (MAF) between Jewish controls and controls from the other cohorts: the MAF for SNP rs13119723 in Jewish controls was 0.06, while the MAF in the other cohorts was between 0.16 and 0.17 . We performed a meta analysis of all CD cohorts including the Jewish cohort (data not shown), which yielded a P value of $1.4 \times 10^{-3}$ for SNP rs 13151961 , a P value of $1.0 \times 10^{-4}$ for SNP rs 13119723, a P value of $4.1 \times 10^{-4}$ for SNP rs6822844 and a P value of $1.4 \times 10^{-3}$ for SNP rs6840978.

Because the association of the IL2/IL21 locus to CD is much more moderate than that to UC it might be that the association is mainly to colonic disease. If this were the case, then we would predict that the association signal from CD comes exclusively from disease localised in the colon. To test this hypothesis we performed a within-cases analysis for the association in colonic and non-colonic CD. However this did not yield any significant results. Further genotype-phenotype analysis for disease localization or extent, disease behaviour, necessity for operation, the occurence of malignancy and extraintestinal manifestations did not yield any phenotype-specific associations (data not shown). Although phenotype data was available for a large proportion of cases ( $80 \%$ for both $\mathrm{CD}$ and $\mathrm{UC}$ ) this might still be due to a lack of power in each specific subgroup to detect true genotype-phenotype associations. 
Another possible explanation for the comparatively modest association to $\mathrm{CD}$ is the relatively low total number of CD patients: 1588 CD patients compared to 3194 UC patients. This, however, does not appear likely as the power calculations showed that with the $1588 \mathrm{CD}$ patients we have in our study there is $95 \%$ power to detect an effect with an OR of 0.85 which is similar to that observed in UC.

\section{Discussion}

In the current study we have identified and replicated a novel association between genetic variants in the IL2/IL2I locus and IBD (OR 0.66; P value $1.4 \times 10^{-9}$ ), with the strongest evidence of association in UC (OR 0.62 ; p-value $1.35 \times 10^{-10}$ ). This association is consistent with the recent findings of a common protective allele in celiac disease, rheumatoid arthritis, psoriasis and type 1 diabetes and thus confirms this locus as a general risk locus for inflammatory disease. $[6,8-10]$

This locus on chromosome $4 \mathrm{q} 27$ comprises a region of $480 \mathrm{~kb}$ of extensive linkage disequilibrium (LD) that harbours the testis nuclear RNA-binding protein (TENR) gene, a gene encoding a protein of unknown function (KIAA1109), and genes encoding the interleukin-2 (IL2) and interleukin-21 (IL21) cytokines. TENR is expressed primarily in testis and KIAA1 109 transcripts are ubiquitous, hence their roles in inflammatory diseases are not particularly compelling, which leaves $I L 2$ and $I L 21$ as the most likely candidates for disease association in the region.[6] As previously reported in other immune diseases, the four SNPs tested and found to be associated to IBD in this study are correlated to each (with $\mathrm{r}^{2}$ correlation coefficients ranging from 0.5 to 0.97 ) and are all located in non-coding regions within this 480 $\mathrm{kb}$ LD block. Two SNPs, rs13151961 and rs13119723, are situated in intronic regions of the KIAA1 109 gene. SNP rs6822844 is located in the intergenic region between IL2 and IL2I and SNP rs6840987 is located downstream of IL21. These SNPs are not known to have an effect on expression of the genes in the IL2-IL21 region.[25]

Interleukin 2 is secreted in an autocrine fashion by antigen-stimulated $\mathrm{T}$ cells and stimulates $\mathrm{T}$ cell activation and proliferation. In these T cells, IL2 stimulates the production of the proinflammatory cytokines interferon-gamma and interleukin 4. Furthermore, IL2 has an important role in regulating the adaptive immune response by stimulating T regulatory (CD4 $+\mathrm{CD} 25+)$ cells and by its ability to stimulate activation-induced cell death in antigen-activated T cells. [26] Interleukin 21 is also a $T$ cell derived cytokine; it stimulates class switching to $\operatorname{IgG}$ in B cells and regulates natural killer cell proliferation and differentiation. IL21 augments proliferation in cells of the monocyte-macrophage lineages and induces an immunosuppressive phenotype by stimulating the formation of immature monocytes that inhibit antigen-specific T cell proliferation. During inflammatory processes, the receptor for interleukin 21, IL-21 R, can be found on non-immune cells, such as colon epithelial cells or fibroblasts. When stimulated by IL-21, these cells secrete proteins that mobilize $\mathrm{T}$ cells to areas of immune challenge.[27]

The overexpression of IL21 in IBD patients compared to healthy controls and diverticulitis patients shows the importance of this interleukin in the inflammatory process of both CD and UC.[17] Interestingly, Monteleone et al. observed the increase in IL21 expression level predominantly in the CD subgroup of IBD patients, whereas we here observed a stronger association of the $I L 2 / I L 21$ locus to UC rather than CD. Although speculative this prioritizes the IL2 gene as the more likely involved gene. IL2 is an attractive functional candidate gene for UC pathogenesis, as the $I l 2-/-$ mouse develops a disease similar to UC, supporting an association between IL2 and UC.[18] The fact that calcineurin inhibitors, which mainly suppress the expression of IL2, are effective in therapy-resistant UC, but not in $\mathrm{CD}$, might also point to a key role of this interleukin in UC.[28] Further support of the importance of IL2 in 
UC comes from the fact that a pilot trial with antibodies against the IL2 receptor in therapyresistant UC was successful.[29] The fact that both a lack of IL2 and an excess of IL2 predispose to colitis is however puzzling. Further functional studies on these genetic variants are needed to define the specific role of the IL2/IL21 locus in the pathogenesis of inflammatory bowel diseases.

An equivalent protective association signal of the IL2/IL2I locus to celiac disease, rheumatoid arthritis, type 1 diabetes and psoriasis has previously been reported. This shows that this locus plays an important role in inflammatory diseases. Previously MAGI2, PARD3, MYOIXB and IL18RAPwere reported to be associated to both celiac disease and UC.[151630-32] The IL2IL21 locus is now the fifth locus to be associated with both diseases, further supporting a model where a common set of biological pathways lead to celiac disease and UC. Interestingly, multiple SNPs in this same region, that are independent of the SNPs studied herein, have recently been reported to confer risk to T1D and potentially to celiac disease.[11] Although these SNPs conferring risk were not tested in the current study a published study in CD $\left(\right.$ rs 17388568, pvalue $=1.7 \times 10^{-4} ;$ rs 716501 , pvalue $=3.8 \times 10^{-4}$ potentially supports the presence of alleles conferring increased risk to disease.[3] Further examination of these riskconferring alleles are warranted in CD and UC.

Extensive sequencing in celiac cases and matched controls, as well as functional studies will be needed to find the true causal variant in the IL2/IL21 locus and determine the molecular mechanisms by which this locus influences an individual's risk to multiple immune mediated diseases.

\section{Supplementary Material}

Refer to Web version on PubMed Central for supplementary material.

\section{References}

1. Baumgart DC, Carding SR. Inflammatory bowel disease: cause and immunobiology. Lancet 2007;369 (9573):1627-1640. [PubMed: 17499605]

2. Baumgart DC, Sandborn WJ. Inflammatory bowel disease: clinical aspects and established and evolving therapies. Lancet 2007;369(9573):1641-1657. [PubMed: 17499606]

3. Barrett JC, Hansoul S, Nicolae DL, et al. Genome-wide association defines more than 30 distinct susceptibility loci for Crohn';s disease. Nat Genet 2008;40:955-962. [PubMed: 18587394]

4. Franke A, Balschun T, Karlsen TH, et al. Sequence variants in IL10, ARPC2 and multiple other loci contribute to ulcerative colitis susceptibility. Nat Genet. 2008

5. Silverberg MS, Cho JH, Rioux JD, et al. Ulcerative colitis-linked loci on chromosome 1p36 and 12q15 found by genome-wide association study. Nat Genet. In press

6. van Heel DA, Franke L, Hunt KA, et al. A genome-wide association study for celiac disease identifies risk variants in the region harboring IL2 and IL21. Nat Genet 2007;39(7):827-829. [PubMed: 17558408]

7. Todd JA, Walker NM, Cooper JD, et al. Robust associations of four new chromosome regions from genome-wide analyses of type 1 diabetes. Nat Genet 2007;39(7):857-864. [PubMed: 17554260]

8. Zhernakova A, Alizadeh BZ, Bevova M, et al. Novel association in chromosome 4q27 region with rheumatoid arthritis and confirmation of type 1 diabetes point to a general risk locus for autoimmune diseases. Am J Hum Genet 2007;81(6):1284-1288. [PubMed: 17999365]

9. Sawalha AH, Kaufman KM, Kelly JA, et al. Genetic association of interleukin-21 polymorphisms with systemic lupus erythematosus. Ann Rheum Dis 2008;67(4):458-461. [PubMed: 17720724]

10. Liu Y, Helms C, Liao W, et al. A genome-wide association study of psoriasis and psoriatic arthritis identifies new disease loci. PLoS Genet 2008;4(3):e1000041. [PubMed: 18369459] 
11. Smyth DJ, Plagnol V, Walker NM, et al. Shared and distinct genetic variants in type 1 diabetes and celiac disease. N Engl J Med 2008;359(26):2767-2777. [PubMed: 19073967]

12. Sanchez E, Morales S, Paco L, et al. Interleukin 12 (IL12B), interleukin 12 receptor (IL12RB1) and interleukin 23 (IL23A) gene polymorphism in systemic lupus erythematosus. Rheumatology (Oxford) 2005;44(9):1136-1139. [PubMed: 15941730]

13. Randolph AG, Lange C, Silverman EK, et al. The IL12B gene is associated with asthma. Am J Hum Genet 2004;75(4):709-715. [PubMed: 15322986]

14. Fisher SA, Tremelling M, Anderson CA, et al. Genetic determinants of ulcerative colitis include the ECM1 locus and five loci implicated in Crohn's disease. Nat Genet 2008;40(6):710-712. [PubMed: 18438406]

15. Hunt KA, Zhernakova A, Turner G, et al. Newly identified genetic risk variants for celiac disease related to the immune response. Nat Genet 2008;40(4):395-402. [PubMed: 18311140]

16. Zhernakova A, Festen EM, Franke L, et al. Genetic analysis of innate immunity in Crohn's disease and ulcerative colitis identifies two susceptibility loci harboring CARD9 and IL18RAP. Am J Hum Genet 2008;82(5):1202-1210. [PubMed: 18439550]

17. Monteleone G, Monteleone I, Fina D, et al. Interleukin-21 enhances T-helper cell type I signaling and interferon-gamma production in Crohn's disease. Gastroenterology 2005;128(3):687-694. [PubMed: 15765404]

18. Sadlack B, Merz H, Schorle H, et al. Ulcerative colitis-like disease in mice with a disrupted interleukin-2 gene. Cell 1993;75(2):253-261. [PubMed: 8402910]

19. Weersma RK, Stokkers PC, van Bodegraven AA, et al. Molecular prediction of disease risk and severity in a large Dutch Crohn's disease cohort. Gut. 2008

20. Duerr RH, Taylor KD, Brant SR, et al. A genome-wide association study identifies IL23R as an inflammatory bowel disease gene. Science 2006;314(5804):1461-1463. [PubMed: 17068223]

21. Rioux JD, Xavier RJ, Taylor KD, et al. Genome-wide association study identifies new susceptibility loci for Crohn disease and implicates autophagy in disease pathogenesis. Nat Genet 2007;39(5):596604. [PubMed: 17435756]

22. Tello-Ruiz MK, Curley C, DelMonte T, et al. Haplotype-based association analysis of 56 functional candidate genes in the IBD6 locus on chromosome 19. Eur J Hum Gene 2006;14(6):780-790.

23. De Jager PL, Franchimont D, Waliszewska A, et al. The role of the Toll receptor pathway in susceptibility to inflammatory bowel diseases. Genes Immun 2007;8(5):387-397. [PubMed: 17538633]

24. Purcell S, Cherny SS, Sham PC. Genetic Power Calculator: design of linkage and association genetic mapping studies of complex traits. Bioinformatics 2003;19(1):149-150. [PubMed: 12499305]

25. Dixon AL, Liang L, Moffatt MF, et al. A genome-wide association study of global gene expression. Nat Genet 2007;39(10):1202-1207. [PubMed: 17873877]

26. Thornton AM, Donovan EE, Piccirillo CA, et al. Cutting edge: IL-2 is critically required for the in vitro activation of CD4+CD25+ T cell suppressor function. J Immunol 2004;172(11):6519-6523. [PubMed: 15153463]

27. Leonard WJ, Spolski R. Interleukin-21: a modulator of lymphoid proliferation, apoptosis and differentiation. Nat Rev Immunol 2005;5(9):688-698. [PubMed: 16138102]

28. Lichtiger S, Present DH, Kornbluth A, et al. Cyclosporine in severe ulcerative colitis refractory to steroid therapy. N Engl J Med 1994;330(26):1841-1845. [PubMed: 8196726]

29. Van Assche G, Dalle I, Noman M, et al. A pilot study on the use of the humanized anti-interleukin-2 receptor antibody daclizumab in active ulcerative colitis. Am J Gastroenterol 2003;98(2):369-376. [PubMed: 12591057]

30. Wapenaar MC, Monsuur AJ, van Bodegraven AA, et al. Associations with tight junction genes PARD3 and MAGI2 in Dutch patients point to a common barrier defect for coeliac disease and ulcerative colitis. Gut 2008;57(4):463-467. [PubMed: 17989107]

31. Monsuur AJ, de Bakker PI, Alizadeh BZ, et al. Myosin IXB variant increases the risk of celiac disease and points toward a primary intestinal barrier defect. Nat Genet 2005;37(12):1341-1344. [PubMed: 16282976]

32. van Bodegraven AA, Curley CR, Hunt KA, et al. Genetic variation in myosin IXB is associated with ulcerative colitis. Gastroenterology 2006;131(6):1768-1774. [PubMed: 17087940] 
ב্

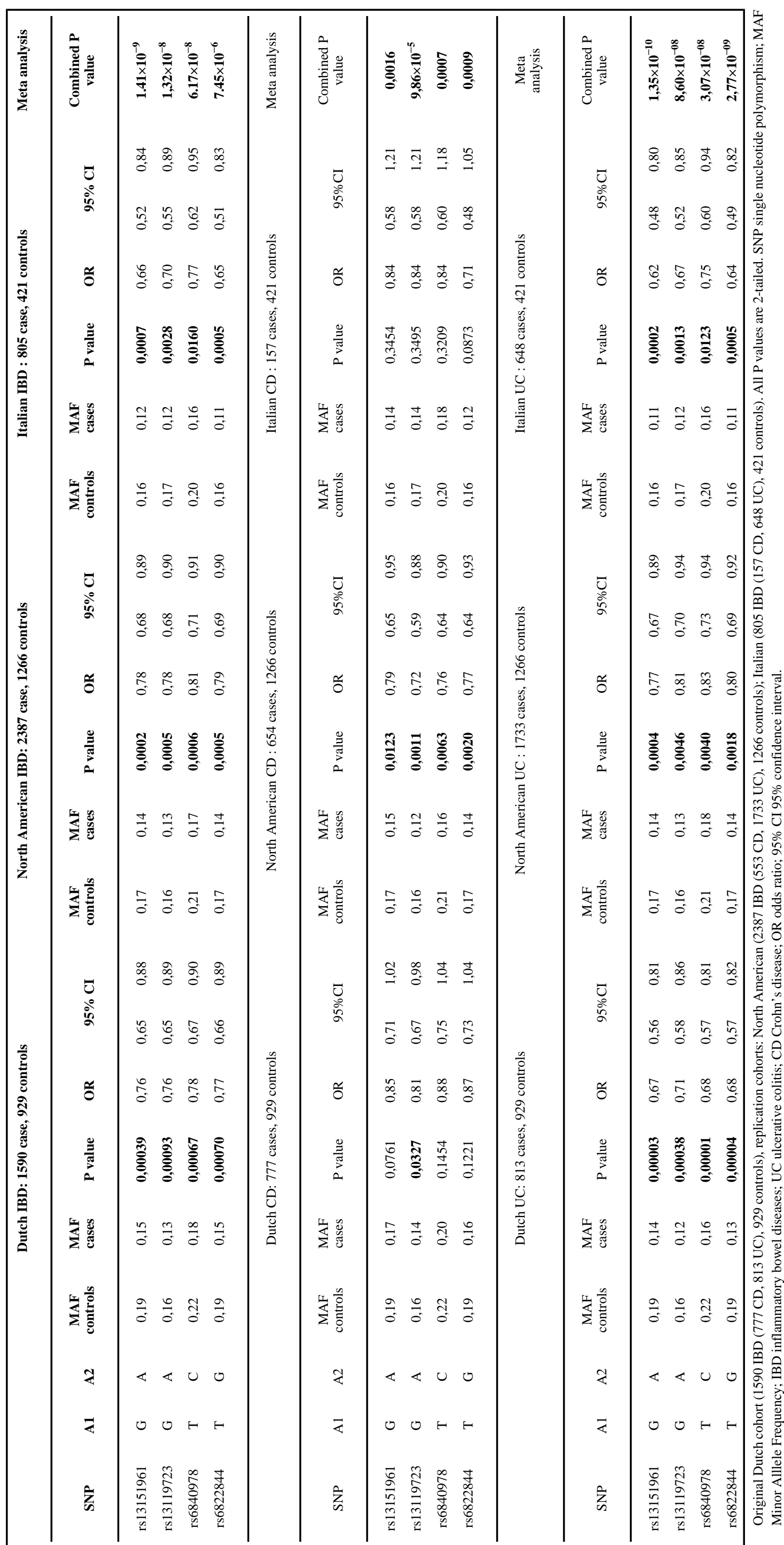


Festen et al.

Page 11

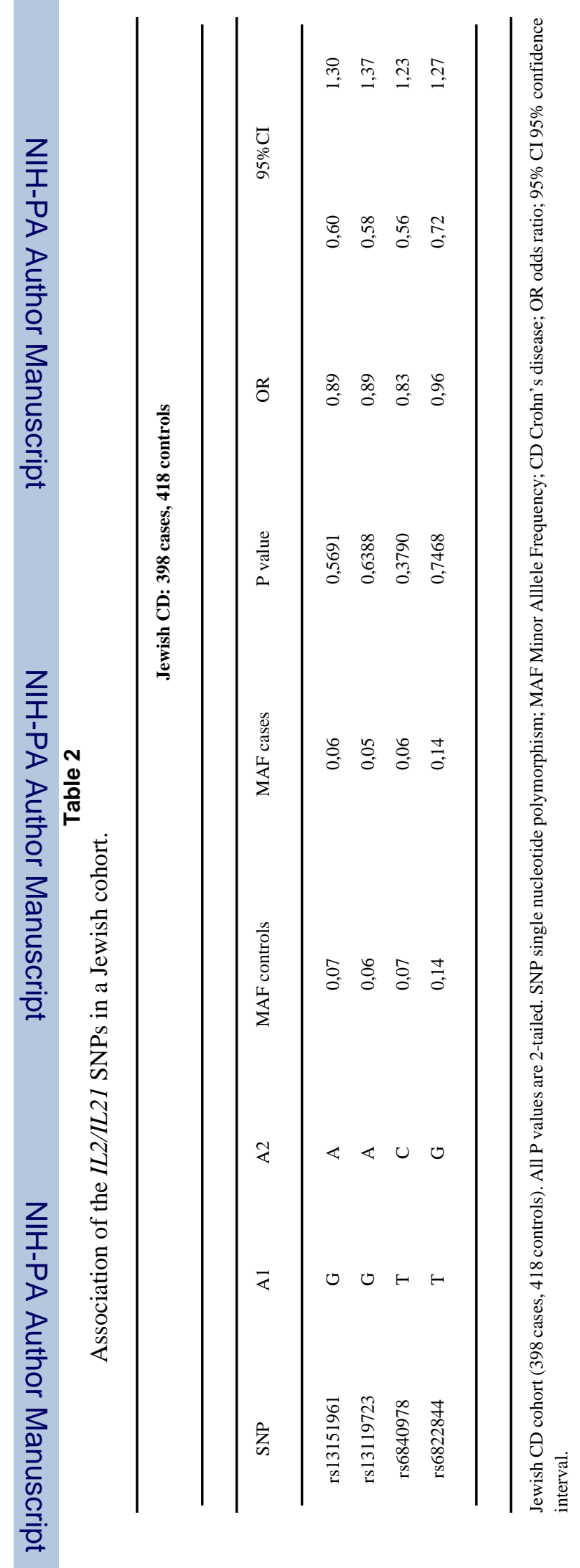

Gut. Author manuscript; available in PMC 2010 June 1. 